\title{
Erratum
}

\section{Synthesis of Selected Novel Covalently Linked Flavoquinolones}

Ram Singh,* Geetanjali Synthesis 2005, 2315.

The author list for this manuscript was incomplete. The name of Professor S. M. S. Chauhan was inadvertently omitted. The correct author list is as follows:

\section{Synthesis of Selected Novel Covalently Linked Flavoquinolones}

R. Singh,* Geetanjali, S. M. S. Chauhan*

Department of Chemistry, University of Delhi, Delhi-110 007, India

E-mail: singh_dr_ram@yahoo.com, smschauhan@chemistry.du.ac.in 